\title{
XXXVII. On the number of fractions contained in any "Farey series" of which the limiting number is given
}

\section{J.J. Sylvester}

To cite this article: J.J. Sylvester (1883) XXXVII. On the number of fractions contained in any "Farey series" of which the limiting number is given , Philosophical Magazine Series 5, 15:94, 251-257, DOI: $10.1080 / 14786448308627346$

To link to this article: http://dx.doi.org/10.1080/14786448308627346

$$
\text { 曲 Published online: } 08 \text { Jun } 2010 .
$$

Submit your article to this journal $\sqsubset$

Џلl Article views: 2

Q View related articles ¿

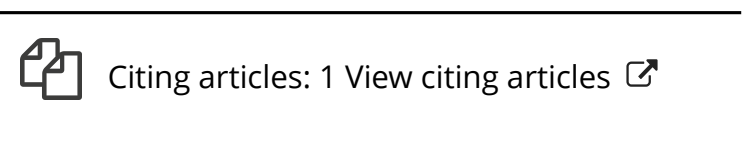


We may here observe (though this is a different point) that the expressions "time-integral of force," for impulsion, and "space-integral of force," for potential energy, have in some cases, from frequent use, reacted upon the users of them; so that we find impulsion called the "total force in a finite time," and potential energy the "sum of the tensions," "force" and "tensions" being force and forces proper. The competent writers now alluded to have inadvertently allowed themselves to use expressions precisely analogous to the statement that the area of a plane curve is the sum of the ordinates. It can be only in some transcendental sense that a force or pressure, supposed constant for simplicity, which has existed statically for a given time, may be said to be the sum of the applications of force in each second of that time; and this is equally true if the force have been existing kinetically.

What we may call the mathematical analogy between energy, or $\mathrm{Fs}$, and impulsion, or $\mathrm{F} t$, is complete, notwithstanding the metaphysical considerations involved in the difference between their factors $s$ and $t$. Each is of two dimensions; while force, or $\mathrm{F}$, is of one. $\mathrm{F}$ being measurable in pounds, $\mathrm{Fs}$ is measurable in foot-pounds, and $\mathrm{F} t$ in secondpounds. Each is equally disparate from $\mathrm{F}$; though very dissimilarly so, since they are disparate from each other. As $\mathrm{F} s$ is the power of performing work, so $\mathrm{F} t$ is the power of producing momentum. Neither bad a name until the present century. It was this that gave room to the remarkable controversy concerning the true measure of the "moving force" of a body in motion which went on for over forty years in the last century, "to the great scandal of science" as Montucla says. Each needs a distinctive appropriated name, as much as the other. One, viz. $\mathrm{Ft}$, has not yet acquired a universally recognized proper name; but the sooner it does so the better for the learner, and also for the science of dynamics, which then need not be guilty of the rather unscientific proceeding of occasionally giving more than one meaning to "Force."

Your obedient servant,

M. H. Close.

XXXVII. On the Number of Fractions contained in any "Farey Series" of which the Limiting Number is given. By J. J. SYLVESTER*

\footnotetext{
A FAREY series ("suite de Farey") is a system of all A the unequal vulgar fractions arranged in order of magnitude, the numerator and denominator of which do not exceed a given number.

The first scientific notice of these series appeared in the

* Communicated by the Author.
} 
Philosophical Magazine, vol. xlvii. (1816) pp. 385, 386. In $1879 \mathrm{Mr}$. Glaisher published in the Philosophical Magazine (pp. 321-336) a paper on the same subject containing a proof of their known properties, an important extension of the subject to series in which the numerators and denominators are subject to distinct limits, and a bibliography of Mr. Goodwyn's tables of such series. Finally, in 1881 Sir George Airy contributed a paper also to the Philosophical Magazine of that year, in which he refers to a table calculated by him "some years ago," and printed in the Selected Papers of the Transactions of the Institution of Civil Engineers, which is in fact a Farey table with the logarithms of the fractions appended to each of them. Previous tables had only given the decimal values of such fractions. The drift of this paper is to point out a caution which is necessary to observe in the use of such tables, and which limits their practical utility : this arises from the fact of the differences receiving a very large augmentation in the immediate neighbourhood of the fractions which are a small aliquot part of unity-a fact which may be inferred $\grave{a}$ priori from the well-known law discovered by Farey applicable to those differences, but to which the author of the paper makes no allusion.

In addition to the tables of Farey series by Goodwyn, Wucherer, an anonymous anthor mentioned in the Babbage Catalogue, and Gauss, referred to by $\mathrm{Mr}$. Glaisher in his Report to the Bradford Meeting of the British Association (1873), may be mentioned one contained in Herzer's Tabellen (Basle, 1864) with the limit 57, and another in Hrabak's Tabellen-Werk (Leipsic, 1876), in which the limit is taken at 50.

The writers on the theory are:-Cauchy (as mentioned by Mr. Glaisher), who inserted a communication relating to it in the Bulletin des Sciences par la Société Philomathique de Paris, republished in his Exercices de Mathématiques ; Mr. Glaisher himself (loc. cit.); M. Halphen, in a recent volume of the Proceedings of the Mathematical Society of France; and M. Lucas, in the next following volume of the same collection. I am indebted to my friend and associate Dr. Story for these later references.

For theoretical purposes it is desirable to count 1 as one of the fractions in a Farey series. The number of such fractions for the limit $j$ then becomes identical with the sum of the totients of all the natural numbers up to $j$ inclusive-a totient to $x$ (which I always denote by $\tau x$ ) meaning the number of numbers less than $x$ and prime to it. Such sum, $i . e$. $x=0$ $\sum_{x=j}^{x=0} \tau x, \mathrm{I}$ denote by $\mathrm{T} j . \quad \mathrm{My}$ attention was called to the subject by this number $\mathrm{T} j$ expressing the number of terms in a 
function whose residue (in Cauchy's sense) is the generating function to any given simple denumerant (see footnote to American Journal of Mathematics, vol. v. p. 123); and I became curious to know something about the value of $\mathrm{T} j$. I had no difficulty in finding a functional equation which serves to determine its limits (see Johns Hopkins University Circular, Jan. and Feb. 1883). The most simple form of that equation (omitted to be given in the Circular) is

$$
\mathrm{T} j+\mathrm{T} \frac{j}{2}+\mathrm{T} \frac{j}{3}+\mathrm{T} \frac{j}{4}+\mathrm{T}_{\overline{5}}^{j}+\ldots=\frac{j^{2}+j}{2},
$$

(where, when $x$ is a fraction, Tx is to be understood to mean $\mathrm{T} j, j$ being the integer next below $x$ ); and from this it is not difficult to deduce by strict demonstration that $\mathrm{T} j / j^{2}$, when $j$ increases indefinitely, approximates indefinitely near to $3 / \pi^{2}$.

I have subsequently found that if $u x$ be used to denote the sum of all the numbers inferior and prime to $x$, and $\mathrm{U}_{j}=\sum_{z=j}^{x=0} u x$,

$$
\mathrm{U} j+2 \mathrm{U} \frac{j}{2}+3 \mathrm{U} \frac{j}{3}+4 \mathrm{U} \frac{j}{4}+\ldots=\frac{j(j+1)(j+2)}{3}
$$

(where $\mathrm{U} x$, when $x$ is a fraction, means the $\mathrm{U}$ of the integer next inferior to $x$ ). From this equation it is also possible to prove that $\mathrm{U} j / j^{3}$, when $j$ becomes indefinitely great, approximates to $1 / \pi^{2}$. $\mathrm{U} j$, it may be well to notice, is the sum of all the numerators of the fractions in a Farey series whose limit is $j$, just as $\mathrm{T} j$ is the number of these fractions.

In the annexed Table the value of $\tau x$ (the totient), of $\mathrm{T} x$ (the sum-totient), and of $3 / \pi^{2} \cdot x^{2}$ is calculated for all the values of $x$ from 1 to 500; and the remarkable fact is brought to light that $\mathrm{T} x$ is always greater than $3 / \pi^{2} \cdot x^{2}$ (the number opposite to it), and less than $3 / \pi^{2} \cdot(x+1)^{2}$, the number which comes after the former one in the same table.

I have calculated in my head the first few values of $\mathrm{U} x$, and find (if I have made no mistake) that it obeys an analogous law, viz. is always intermediate between $1 / \pi^{2} . x^{3}$ and $1 / \pi^{2} .(x+1)^{3}$.

It may also be noticed that when $n$ is a prime number, $\mathrm{T} n$ is always nearer, and usually very much nearer, to the superior than to the inferior limit-as might have been anticipated from the circumstance that, when this is the case, in passing from $n-1$ to $n$ the $\mathrm{T}$ receives an augmentation of $n-1$, whereas its average augmentation is only $\frac{3}{\pi^{2}}(2 n-1)$.

In like manner and for a similar reason, when $n$ contains several small factors $\mathrm{T} n$ is nearer to the inferior than to the superior limit. For instance, when $n=210, \mathrm{~T} n=13414$ and $3 / \pi^{2} . n^{2}=1340479$. 
TABLE of Totients, of Sum-totients, and of $3 / \pi^{2}$ into the Squares of all the Numbers from 1 to 500 inclusive.

$$
\left[\frac{3}{\pi^{2}}=\cdot 30396355\right] \text {. }
$$

\begin{tabular}{|c|c|c|c|c|c|c|c|c|c|c|c|}
\hline$n$ & $r(n)$ & $\mathbf{T}(n)$ & $\frac{3}{\pi^{2}} n^{2}$ & $n$ & $\tau(n)$ & $T(n)$ & $\frac{3}{\pi^{2}} n^{2}$ & $n$ & $r(n)$ & $\mathrm{T}(n)$ & $\frac{3}{\pi^{2}} n^{2}$ \\
\hline 1 & 1 & 1 & 30 & 49 & 42 & 754 & $729 \cdot 82$ & 97 & 96 & 2902 & $2860 \cdot 00$ \\
\hline 2 & 1 & 2 & $1 \cdot 22$ & 50 & 20 & 774 & $759 \cdot 91$ & 98 & 42 & 2944 & 291927 \\
\hline 3 & 2 & 4 & 274 & 51 & 32 & 806 & $790 \cdot 61$ & 99 & 60 & 3004 & $2979 \cdot 15$ \\
\hline 4 & 2 & 6 & 4.86 & 52 & 24 & 830 & $8: 1 \cdot 92$ & 100 & 40 & 3044 & $3039 \cdot 64$ \\
\hline 5 & 4 & 10 & $7 \cdot 60$ & 53 & 52 & 882 & $853 \cdot 83$ & 101 & 100 & 3144 & 310073 \\
\hline 6 & 2 & 12 & $10 \cdot 94$ & 54 & 18 & 900 & 88636 & 102 & 32 & 3176 & $3162 \cdot 44$ \\
\hline 7 & 6 & 18 & 14.90 & 55 & 40 & 940 & $919 \cdot 49$ & 103 & 102 & 3278 & $3224 \cdot 75$ \\
\hline 8 & 4 & 22 & $19 \cdot 46$ & 56 & 24 & 964 & 95323 & 104 & 48 & 3326 & $3287 \cdot 67$ \\
\hline 9 & 6 & 28 & 2462 & 57 & 36 & 1000 & 987.58 & 105 & 48 & 3374 & $3351 \cdot 20$ \\
\hline 10 & 4 & 32 & $30 \cdot 40$ & 58 & 28 & 1028 & $1022 \cdot 54$ & 106 & 52 & 3426 & 341534 \\
\hline 11 & 10 & 42 & $36 \cdot 78$ & 59 & 58 & 1086 & $1058 \cdot 10$ & 107 & 106 & 3532 & $3480 \cdot 08$ \\
\hline 12 & 4 & 46 & $43 \cdot 77$ & 60 & 16 & 1102 & $1094 \cdot 27$ & 108 & 36 & 3568 & $3545 \cdot 44$ \\
\hline 13 & 12 & 58 & $51 \cdot 37$ & 61 & 60 & 1162 & $1131 \cdot 05$ & 109 & 108 & 3676 & $3611 \cdot 40$ \\
\hline 14 & 6 & 64 & $59 \cdot 58$ & 62 & 30 & 1192 & $1168 \cdot 44$ & 110 & 40 & 3716 & $3677 \cdot 96$ \\
\hline 15 & 8 & 72 & $68: 39$ & 63 & 36 & 1228 & $1206 \cdot 43$ & 111 & 72 & 3788 & $3745 \cdot 14$ \\
\hline 16 & 8 & 80 & 77.81 & 64 & 32 & 1.260 & 1245.03 & 112 & 48 & 3836 & $3812 \cdot 92$ \\
\hline 17 & 16 & 96 & $87 \cdot 84$ & 65 & 48 & 1308 & $1284 \cdot 25$ & 113 & 112 & 3948 & 388131 \\
\hline 18 & 6 & 102 & $98 \cdot 48$ & $66^{\circ}$ & 20 & 1328 & $1324 \cdot 07$ & 114 & 36 & 3984 & $3950 \cdot 31$ \\
\hline 19 & 18 & 120 & $109 \cdot 73$ & 67 & 66 & 1394 & $1364 \cdot 49$ & 115 & 88 & 4072 & $4019 \cdot 92$ \\
\hline 20 & 8 & 128 & $121 \cdot 58$ & 68 & 32 & 1426 & 1405.53 & 116 & 56 & 4128 & $4090 \cdot 14$ \\
\hline 21 & 12 & 140 & 134.05 & 69 & 44 & 1470 & $1447 \cdot 17$ & 117 & 72 & 4200 & 4160.96 \\
\hline 22 & 10 & 150 & $147 \cdot 12$ & 70 & 24 & 1494 & $1489 \cdot 42$ & 118 & 58 & 4258 & $4232 \cdot 39$ \\
\hline 23 & 22 & 172 & $160 \cdot 79$ & 71 & 70 & 1564 & $1532 \cdot 28$ & 119 & 96 & 4354 & $4304 \cdot 43$ \\
\hline 24 & 8 & 180 & $175 \cdot 08$ & 72 & 24 & 1588 & 157575 & 120 & 32 & 4386 & $4377 \cdot 08$ \\
\hline 25 & 20 & 200 & $189 \cdot 98$ & 73 & 72 & 1660 & 1619.82 & 121 & 110 & 4496 & $4450 \cdot 33$ \\
\hline 26 & 12 & 212 & 5.48 & 74 & 36 & 1696 & $1664 \cdot 51$ & 122 & 60 & 4556 & $4524 \cdot 19$ \\
\hline 27 & 18 & 230 & 221.59 & 75 & 40 & 1736 & $1709 \cdot 80$ & 123 & 80 & 4636 & $4598 \cdot 66$ \\
\hline 28 & 12 & 242 & $238 \cdot 31$ & 76 & 36 & 1772 & 175569 & 124 & 60 & 4696 & $4673 \cdot 74$ \\
\hline 29 & 28 & 270 & $25 \overline{5} \cdot 63$ & 77 & 60 & 1832 & $1802 \cdot 20$ & 125 & 100 & 4796 & $4749 \cdot 43$ \\
\hline 30 & 8 & 278 & $273 \cdot 56$ & 78 & 24 & 1856 & $1849 \cdot 31$ & 126 & 36 & 4832 & $4825 \cdot 72$ \\
\hline 31 & 30 & 308 & $292 \cdot 11$ & 79 & 78 & 1934 & 1897.04 & 127 & 126 & 4958 & $4902 \cdot 63$ \\
\hline 32 & 16 & 324 & $311 \cdot 26$ & 80 & 32 & 1966 & $1945 \cdot 37$ & 128 & 64 & 5022 & $4980 \cdot 14$ \\
\hline 33 & 20 & 344 & 333.01 & 81 & 54 & 2020 & & 129 & 84 & 5106 & $5058 \cdot 26$ \\
\hline 34 & 16 & 360 & $351 \cdot 38$ & 82 & 40 & 3060 & $2043 \cdot 85$ & 130 & 48 & 5154 & $5136 \cdot 98$ \\
\hline 35 & 24 & 384 & 37235 & 83 & 82 & 2142 & $2094 \cdot 01$ & 131 & 130 & 5284 & 521632 \\
\hline 36 & 12 & 396 & 393.93 & 84 & 24 & 2166 & $2144 \cdot 77$ & 132 & 40 & 5324 & $5296 \cdot 26$ \\
\hline 37 & 36 & 432 & $416 \cdot 12$ & 85 & 64 & 2230 & $2196-14$ & 133 & 108 & 5432 & 537681 \\
\hline 38 & 18 & 450 & & $86^{\circ}$ & 42 & 2272 & & 134 & 66 & 5498 & $5457 \cdot 97$ \\
\hline 39 & 24 & 474 & $462 \cdot 32$ & 87 & 56 & 2328 & $2300 \cdot 70$ & 135 & 72 & 5570 & $5539 \cdot 74$ \\
\hline 40 & 16 & 490 & $486 \cdot 34$ & 88 & 40 & 2368 & $2353 \cdot 90$ & 136 & 64 & 5634 & $5622 \cdot 11$ \\
\hline 41 & 40 & 530 & $510 \cdot 96$ & 89 & 88 & 2456 & $2407 \cdot 70$ & 137 & 136 & 5770 & $5705 \cdot 09$ \\
\hline 42 & 12 & 542 & $536 \cdot 19$ & 90 & 24 & 2480 & $2462 \cdot 10$ & 138 & 44 & 5814 & $5788 \cdot 68$ \\
\hline 43 & 42 & 584 & $562 \cdot 02$ & 91 & 72 & 2552 & $2517 \cdot 12$ & 139 & 138 & 5952 & $5872 \cdot 88$ \\
\hline 44 & 20 & 604 & $588 \cdot 47$ & 92 & 44 & 2596 & $2572 \cdot 75$ & 140 & 48 & 6000 & 595769 \\
\hline 45 & 24 & 628 & $615 . \tilde{5} 2$ & 93 & 60 & 2656 & $2628 \cdot 8$ & 141 & 92 & 6092 & $6043 \cdot 10$ \\
\hline 46 & 22 & 650 & $643 \cdot 19$ & 94 & 46 & 2702 & 2685.82 & 142 & 70 & 6162 & $6129 \cdot 12$ \\
\hline 47 & 46 & 696 & $671 \cdot 45$ & 95 & 72 & 2774 & $2743 \cdot 27$ & 143 & 120 & 6282 & $6215 \cdot 75$ \\
\hline 48 & 16 & 712 & $700 \cdot 33$ & 96 & 32 & 2806 & $2801 \cdot 33$ & 144 & 48 & 6330 & $6302: 99$ \\
\hline
\end{tabular}


Table (continued).

\begin{tabular}{|c|c|c|c|c|c|c|c|c|c|c|c|}
\hline$n$ & $\tau(n)$ & $T(n)$ & $\frac{3}{\pi^{2}} n^{2}$ & $n$ & $\tau(n)$ & $\mathbf{T}(n)$ & $\frac{3}{\pi^{2}} n^{2}$ & $n$ & $\tau(n)$ & $\mathbf{T}(n)$ & $\frac{3}{\pi^{2}} n^{2}$ \\
\hline 145 & 112 & 6442 & $6390 \cdot 83$ & 198 & 60 & & 11916.59 & 251 & 250 & 19274 & \\
\hline & 72 & & $6479 \cdot 29$ & 199 & 198 & 12152 & 1 & 252 & 72 & 19346 & \\
\hline 14 & 84 & 6598 & & 200 & 80 & 12232 & & 253 & 220 & 19566 & \\
\hline & 72 & 6670 & $6658 \cdot 02$ & 201 & 132 & 12364 & $12280^{\circ} 43$ & 254 & 126 & 19692 & 19610.51 \\
\hline 149 & & 6818 & & 202 & 100 & 12464 & $12402 \cdot 93$ & 255 & 128 & 19820 & \\
\hline 150 & 40 & 6858 & & 203 & 168 & 12632 & $12526^{\circ} \cdot 03$ & 256 & 128 & 19948 & $19920 \cdot 56$ \\
\hline 151 & 150 & 7008 & & 204 & 64 & 12696 & $12649 \cdot 75$ & 257 & 256 & & \\
\hline 152 & 72 & 7080 & 702277 & 205 & 160 & 12856 & $1277+07$ & 258 & 84 & 20288 & 20233.03 \\
\hline 153 & 96 & 7176 & & 206 & 102 & & & 259 & 216 & 20504 & $20390 \cdot 18$ \\
\hline 154 & 60 & 7236 & 7208.80 & 207 & 132 & 13090 & $13024 \cdot 54$ & 260 & 96 & 20600 & 20547.94 \\
\hline 15 & 120 & 7356 & $7302 \cdot 72$ & 208 & 96 & 13186 & $13150 \cdot 68$ & 261 & 168 & 20768 & $20706 \cdot 30$ \\
\hline 156 & 48 & 7404 & & 209 & 180 & 13366 & 13277.43 & 262 & 130 & & \\
\hline 157 & 150 & 7560 & $7492 \cdot 40$ & 210 & 48 & 13414 & 1340479 & 263 & 262 & 21160 & 2102486 \\
\hline 158 & 78 & 7638 & & 211 & 210 & & & 264 & 80 & & \\
\hline 159 & 104 & 7742 & & 212 & 104 & 13728 & $1366 \mathrm{l} \cdot 34$ & 265 & 208 & 21448 & $45 \cdot 84$ \\
\hline 160 & 64 & 7806 & & 213 & 140 & 13868 & 137 ? & 266 & 108 & 21556 & $37 \cdot 25$ \\
\hline 161 & 132 & 7938 & & 214 & 106 & 13974 & & 267 & 176 & & \\
\hline 162 & 54 & $79 ! 12$ & & 215 & 168 & 14142 & & 268 & 132 & 21864 & \\
\hline 163 & 162 & 8154 & & 216 & 72 & 142 & & 269 & 268 & & \\
\hline 164 & 80 & 8234 & & 217 & 180 & 143 & 14 & 270 & 72 & 22204 & $8 \cdot 95$ \\
\hline 16.5 & 80 & 8314 & & 218 & 108 & 14 & & 271 & 270 & 2474 & \\
\hline 166 & 82 & 8396 & & 219 & 144 & 140 & & 272 & 128 & 22602 & 44 \\
\hline 167 & 166 & 8562 & & 220 & 80 & 14726 & $\cdot 84$ & 273 & 144 & 22746 & $4 \cdot 10$ \\
\hline 168 & 48 & 8610 & & 221 & 192 & & & 274 & 136 & 22882 & \\
\hline 169 & 156 & 8760 & & 222 & 72 & 148 & & 275 & 200 & 23082 & \\
\hline 170 & 64 & 8830 & 878 & 223 & 222 & 15212 & & 276 & 88 & 23170 & \\
\hline 171 & 108 & 8938 & & 224 & 96 & 15 & & 277 & 276 & & \\
\hline 172 & 84 & 9022 & 899 & 225 & 120 & 154 & & 278 & 138 & 23584 & \\
\hline 173 & 172 & 9194 & & 226 & 112 & & & 279 & 180 & & \\
\hline 174 & 56 & 9250 & & 227 & 226 & 15766 & 156 & 280 & 96 & 23860 & 238 \\
\hline 175 & 120 & 9370 & & 228 & 72 & & & 281 & 280 & 24140 & \\
\hline 176 & 80 & 9450 & & 229 & 228 & 16066 & & 282 & 92 & 24232 & \\
\hline 177 & 116 & 9566 & 952 & 230 & 88 & 16154 & 67 & 283 & 282 & 24514 & 24 \\
\hline 178 & 88 & 96 & & 231 & 120 & & & 284 & 140 & & \\
\hline 179 & 178 & 9832 & 29 & 232 & 112 & 168 & & 280 & 144 & 24798 & \\
\hline 180 & 48 & 988 & & 233 & 232 & & & 286 & 120 & & \\
\hline 181 & 180 & 10060 & & 234 & 72 & & & 287 & 240 & & \\
\hline 182 & 72 & & & 235 & 184 & & & 288 & 96 & & \\
\hline 183 & 120 & & & 236 & 116 & & & 289 & 272 & & \\
\hline 184 & 88 & 10340 & 102 & 237 & 156 & & & 290 & 112 & 25638 & \\
\hline 185 & 144 & 104 & & 238 & 96 & & & 291 & 192 & & \\
\hline 186 & 60 & 10544 & & 239 & 238 & & & 292 & 144 & 25974 & \\
\hline 187 & I 60 & 107 & & 240 & 64 & & & 293 & 292 & & \\
\hline 189 & 92 & & & $24 y$ & 240 & & & 294 & 84 & & \\
\hline 189 & 108 & 1090 & & 242 & 110 & & & 295 & 232 & 26582 & 2645 \\
\hline 190 & 72 & & & 243 & 162 & & & 296 & 144 & & \\
\hline $19 !$ & 190 & & & 244 & 120 & & & 297 & 180 & & \\
\hline 192 & 64 & $\mathrm{~L} 12 \mathrm{~s}$ & $11 \%$ & 245 & 168 & & & 298 & 148 & & \\
\hline 193 & 192 & 114 & & 246 & 80 & & & 299 & 264 & 27318 & 271 \\
\hline 194 & 96 & 115 & & 247 & 216 & & & 300 & 80 & & \\
\hline 195 & 96 & & 115 & 248 & 120 & & & 301 & 252 & 27650 & 2753 \\
\hline 196 & 84 & & 11677 & 249 & 164 & & & 302 & 150 & & \\
\hline 197 & 196 & & 1179652 & 250 & 100 & 190 & 18997.72 & 303 & 200 & & \\
\hline
\end{tabular}




\section{Number of Fractions contained in any "Farey Series."}

\section{Table (continued).}

\begin{tabular}{|c|c|c|c|c|c|c|c|c|c|c|c|}
\hline$n$ & $\boldsymbol{T}(n)$ & $\mathbf{T}(n)$ & $\frac{3}{\pi^{2}} n^{2}$ & $n$ & $\tau(n)$ & $\mathbf{T}(n)$ & $\frac{3}{\pi^{2}} n^{2}$ & $n$ & $\tau(n)$ & $\mathrm{T}(n)$ & $\frac{3}{\pi} n^{2}$ \\
\hline 304 & 144 & 28144 & $28091 \cdot 10$ & 357 & 192 & $38 \$ 22$ & $38739 \cdot 85$ & 410 & 160 & 51210 & $51096 * 7$ \\
\hline 305 & 240 & 384 & $8276 * 21$ & 358 & 178 & 39000 & $38957 \cdot 18$ & 411 & 272 & & \\
\hline 306 & 96 & 28480 & & 359 & 358 & 39358 & 3917513 & 412 & 204 & & \\
\hline 307 & 306 & 28786 & $28648 \cdot 26$ & 360 & 96 & 39454 & $39393 \cdot 68$ & 413 & 348 & 52034 & \\
\hline 308 & 120 & 28906 & $28835 \cdot 20$ & 361 & 342 & 39796 & $39612 \cdot 83$ & 414 & 132 & 52166 & \\
\hline 309 & 204 & 29110 & 2902275 & 362 & 180 & 39976 & $39832 \cdot 60$ & 415 & 328 & 52494 & $52350 \cdot 12$ \\
\hline 310 & 120 & 29230 & $29210 \cdot 90$ & 363 & 220 & 40196 & $40052 \cdot 97$ & 416 & 192 & 52686 & \\
\hline 311 & 310 & 29540 & 2939966 & 364 & 144 & 40340 & 40273.95 & 417 & 276 & 52962 & 5285592 \\
\hline 312 & 96 & 29636 & $29589 \cdot 03$ & 365 & 288 & 40628 & $40495 \cdot 54$ & 418 & 180 & 53142 & $53109 \cdot 73$ \\
\hline 313 & 312 & 29948 & $29779 \cdot 01$ & 366 & 120 & 40748 & $40717 \cdot 74$ & 419 & 418 & 53560 & $53364 \cdot 15$ \\
\hline 314 & 156 & 30104 & 29969.59 & 367 & 366 & 41114 & 40940.55 & 420 & 96 & 53656 & $53619 \cdot 17$ \\
\hline 315 & 144 & 30248 & $30160 \cdot 79$ & 368 & 176 & 41290 & $41163 \cdot 96$ & 421 & 420 & 54076 & 5387480 \\
\hline 316 & 156 & 30404 & $30352 \cdot 59$ & 369 & 240 & 41530 & 41387.98 & 422 & 210 & 54286 & \\
\hline 317 & 316 & 30720 & 3054500 & 370 & 144 & 41674 & $41612 \cdot 61$ & 423 & 276 & 54562 & 54387 \\
\hline 318 & 104 & 30824 & $30738 \cdot 01$ & 371 & 312 & 41986 & $41837 \cdot 85$ & 424 & 208 & 54770 & \\
\hline 319 & 280 & 31104 & $30931 \cdot 64$ & 372 & 120 & 42106 & $42063 \cdot 69$ & 425 & 320 & 55090 & $54903 \cdot 42$ \\
\hline 320 & 128 & 31232 & $31125 \cdot 87$ & 373 & 372 & 42478 & $42290 \cdot 15$ & 426 & 140 & 55230 & $5 \overline{0} 162 \% 09$ \\
\hline 321 & 212 & 31444 & 3132071 & 374 & 160 & 42638 & $42517 \cdot 21$ & 427 & 360 & 55590 & $55421 \cdot 39$ \\
\hline 322 & 132 & 31576 & $31516 \cdot 16$ & 375 & 200 & 42838 & $42744 \cdot 87$ & 428 & 212 & 55802 & $55681 \cdot 26$ \\
\hline 323 & 288 & 31864 & $31712 \cdot 22$ & 376 & 184 & 43022 & $42973 \cdot 15$ & 429 & 240 & 56042 & \\
\hline 324 & 108 & 31972 & $31908 \cdot 88$ & 377 & 336 & 43358 & $43202 \cdot 04$ & 430 & 168 & 56210 & 5620 \\
\hline 325 & 240 & 32212 & $32106 \cdot 15$ & 378 & 108 & 43466 & $43431 \cdot 5$ & 431 & 430 & 56640 & 5646 \\
\hline 326 & 162 & 32374 & $32304 \cdot 03$ & 379 & 378 & 43844 & $43661 \cdot 63$ & 432 & 144 & 56784 & 567 \\
\hline 327 & 216 & 32590 & $3250 \cdot 52$ & 380 & 144 & 43988 & $43892 \cdot 34$ & 433 & 432 & 57216 & 5698 \\
\hline 328 & 160 & 32750 & $32701 \cdot 62$ & 381 & 252 & 44240 & $14123 \cdot 65$ & 434 & 180 & 57396 & \\
\hline 329 & $276^{\circ}$ & 33026 & $32901 \cdot 32$ & 382 & 190 & 44430 & $44355 \cdot 58$ & 435 & 224 & 57620 & 57517.50 \\
\hline 330 & 80 & 33106 & 3310 & 383 & 382 & 44812 & 4458 & 436 & 216 & 57836 & 5778 \\
\hline 331 & 330 & 33436 & $33302 \div 55$ & 384 & 128 & 44940 & $44821 \cdot 25$ & 437 & 396 & 58232 & 5804 \\
\hline 332 & 164 & 33600 & $33504 \cdot 08$ & 385 & 240 & 45180 & $45055 \cdot 00$ & 438 & 144 & 58376 & 5831 \\
\hline & 216 & 33816 & & 386 & 192 & 45372 & 45289 & 439 & 438 & 588 & \\
\hline 334 & 166 & 33982 & 33908.96 & 387 & 252 & 45624 & $45524 \cdot 32$ & 440 & 160 & 58974 & 5884 \\
\hline 335 & 264 & 34246 & & 388 & 192 & 45816 & & 441 & 252 & & \\
\hline 336 & 96 & 34342 & 3431 & 389 & 388 & 46204 & $45996 \cdot 07$ & 442 & 192 & 59418 & 5938 \\
\hline 337 & 336 & 34678 & 345 & 390 & 96 & 46300 & & 443 & 442 & 860 & \\
\hline 338 & 156 & 34834 & $34726 \cdot 01$ & 391 & 352 & 46652 & $46470 \cdot 25$ & 444 & 144 & & \\
\hline 339 & 224 & 35058 & $34931 \cdot 80$ & 392 & 168 & 46820 & $46708 \cdot 25$ & 445 & 352 & 60356 & 60192 \\
\hline 340 & 128 & 35186 & & 393 & 260 & & & $44 \delta$ & 222 & 60578 & \\
\hline 341 & 300 & 35486 & 3534 & 394 & 196 & 47276 & 47186.09 & 447 & 296 & 60874 & 607 \\
\hline 342 & 108 & & 3 & 395 & 312 & 47588 & & 448 & 192 & & \\
\hline 343 & 294 & 35888 & 3 & 396 & 120 & 47708 & 4766 & 449 & 448 & 615 & \\
\hline 3 & 168 & 6056 & & 397 & 396 & 48104 & & 450 & 120 & 61634 & \\
\hline 345 & 176 & 36232 & & 398 & 198 & 48302 & & 451 & 400 & & \\
\hline 34 & 172 & 36404 & & 399 & 216 & $48 \overline{1} 18$ & $48391 \cdot 30$ & 452 & 224 & 62258 & \\
\hline 34 & 346 & & & 400 & 160 & & & 453 & 300 & 62558 & \\
\hline & 112 & 6862 & $36811 \cdot 21$ & 401 & 400 & 49078 & & 454 & 226 & 62784 & \\
\hline 349 & 348 & & & 402 & 132 & 49210 & & 455 & 288 & & \\
\hline & 120 & 7330 & & 403 & 360 & 49570 & & 456 & 144 & & \\
\hline $3 \tilde{3} 1$ & 216 & 37546 & 3744861 & 404 & 200 & 49770 & $49611 \cdot 72$ & 457 & 456 & & \\
\hline & 160 & & 37602 & 405 & 216 & & & 458 & 228 & & \\
\hline & 352 & & & 406 & 168 & & & 459 & 288 & & \\
\hline & 116 & & & 407 & 360 & & & 460 & 176 & & \\
\hline 335 & 280 & & & 408 & 128 & & 50598.99 & 461 & 460 & & \\
\hline 356 & 176 & 38630 & $38523 \cdot 12$ & 409 & 408 & 51050 & $50847 \cdot 33$ & 462 & 120 & 64944 & $64879 \cdot 20$ \\
\hline
\end{tabular}


lable (continued).

\begin{tabular}{|c|c|c|c|c|c|c|c|c|c|c|c|}
\hline$n$ & $\tau(n)$ & $T(n)$ & $\frac{3}{\pi^{2}} n^{2}$ & $n$ & $\tau(n)$ & $\mathrm{T}(n)$ & $\frac{3}{\pi^{2}} n^{2}$ & $n$ & $\tau(n)$ & $\mathrm{T}(n)$ & $\frac{3}{\pi^{2}} n^{2}$ \\
\hline 463 & 462 & 65406 & $65160 \cdot 36$ & 476 & 192 & 68964 & $68870 \cdot 85$ & 489 & 324 & 72872 & $72684-07$ \\
\hline 464 & 224 & 65630 & $65442 \cdot 14$ & 477 & 312 & 69276 & $69160 \cdot 52$ & 490 & 168 & 73040 & $7 \cdot 991 \cdot 65$ \\
\hline 465 & 240 & 65870 & 65724.52 & 478 & 238 & 69514 & $69450-81$ & 491 & 490 & 73530 & 73279.84 \\
\hline 466 & 232 & 66102 & $66007 \cdot 51$ & 479 & 478 & 69992 & $69741 \cdot 70$ & 492 & 160 & 73690 & $73578 \cdot 63$ \\
\hline 467 & 466 & 66568 & $66291 \cdot 11$ & 480 & 128 & 70120 & $70033 \cdot 20$ & 493 & 448 & 74138 & 73878.04 \\
\hline & 144 & 66712 & $66575 \cdot 31$ & 481 & 432 & 70552 & $70325 \cdot 31$ & 494 & 216 & 74354 & 7417805 \\
\hline 469 & 396 & 67108 & $66860 \cdot 13$ & 482 & 240 & 70792 & 70618.03 & 495 & 240 & 74594 & 7447867 \\
\hline 470 & 184 & 67292 & $67145 \cdot 55$ & 483 & 264 & 71056 & 709 & 496 & 240 & 74834 & 74779.90 \\
\hline 471 & 312 & 67604 & & 484 & 220 & 71276 & 71205 & 497 & 420 & 75254 & $75081 \cdot 73$ \\
\hline 472 & 232 & 67836 & $67718 \cdot 22$ & 485 & 384 & 71660 & $71499 \cdot 83$ & 498 & 164 & 75418 & $75384 \cdot 18$ \\
\hline 473 & 420 & 68256 & $68005 \cdot 46$ & 486 & 162 & 71822 & $71794 \cdot 98$ & 499 & 498 & & $75687 \cdot 23$ \\
\hline 474 & 156 & 68412 & 6829332 & 487 & 486 & 72308 & $72090 \cdot 73$ & 500 & 200 & 76116 & $75990-89$ \\
\hline 475 & 360 & 68772 & $68581 \cdot 78$ & 488 & 240 & 72548 & $72387 \cdot 10$ & & & & \\
\hline
\end{tabular}

XXXVIII. On Permanent Magnetism.

By R. H. M. Bosaxquet, St. John's College, Oxford.

To the Editors of the Philosophical Magazine and Journal.

Gentlemen,

T HAVE succeeded in completely determining, to a mode-

1 rate approximation, all the quantities involved in the experiment mentioned in my paper in the March number of the Philosophical Magazine. I propose to give a short summary of the results, reserving a more complete account for a future occasion.

The external or air resistances were determined by magnetizing, in the bifilar, arrangements of soft iron similar to the steel. The resistance of the soft iron is small, and is allowed for by Rowland's table. The total resistance is the quotient of the magnetomotive force, $(4 \pi \mathrm{C} n)$, by the magnetic induction $\mathfrak{B}$.

A set of 18 pieces of soft iron similar to the pieces of the compound magnet gave for the total resistance of each piece -454 centim.

$\mathfrak{B}=116$ gives from Rowland's table $\mu=$ about 400 , whence $\frac{1.58}{400}=.004$ is to be subtracted, and we have for the air resistance 450 centimetre, or $47 \mathrm{R}$. ( $R$ is not quite the same in the iron and steel.)

A long piece of soft iron, similar to the whole magnet joined up, gave

Total resistance $=\cdot 339$ centim. 